University of South Carolina

Scholar Commons

$1-27-1986$

\title{
Direct Observation of Ensemble Averaging of the Aharonov-Bohm Effect in Normal-Metal Loops
}

C. P. Umbach

C. Van Haesendonck

R. B. Laibowitz

S. Washburn

Richard A. Webb

University of South Carolina - Columbia, webbra@mailbox.sc.edu

Follow this and additional works at: https://scholarcommons.sc.edu/phys_facpub

Part of the Physics Commons

Publication Info

Published in Physical Review Letters, ed. Gene D. Sprouse, Volume 56, Issue 4, 1986, pages 386-389.

Umbach, C. P., Van Haesendonck, C., Laibowitz, R. B., Washburn, S., Webb, R. A. (1986). Direct observation of ensemble averaging of the Aharonov-Bohm effect in normal-metal loops. Physical Review Letters, 56(4), 386-389. DOI: 10.1103/PhysRevLett.56.386

(C) Physical Review Letters, 1986, American Physical Society

This Article is brought to you by the Physics and Astronomy, Department of at Scholar Commons. It has been accepted for inclusion in Faculty Publications by an authorized administrator of Scholar Commons. For more information, please contact digres@mailbox.sc.edu. 


\title{
Direct Observation of Ensemble Averaging of the Aharonov-Bohm Effect in Normal-Metal Loops
}

\author{
C. P. Umbach, C. Van Haesendonck, ${ }^{(a)}$ R. B. Laibowitz, S. Washburn, and R. A. Webb \\ IBM T.J. Watson Research Center, Yorktown Heights, New York 10598 \\ (Received 6 November 1985)
}

\begin{abstract}
Aharonov-Bohm magnetoconductance oscillations have been measured in series arrays of one, three, ten, and thirty submicron-diameter Ag loops. At constant temperature, the amplitude of the $h / e$ oscillations is observed to decrease as the square root of the number of loops, while the amplitude of the $h / 2 e$ conductance oscillations, measured in the same samples, is independent of the number of series loops. This is direct confirmation of the ensemble-averaging properties of $h / e$ oscillations in multiloop systems. The amplitude of the $h / e$ oscillations is in good agreement with recent calculations.
\end{abstract}

PACS numbers: $72.15 . \mathrm{Gd}, 73.60 . \mathrm{Dt}$

Flux-periodic quantum corrections to the magnetoconductance of multiply connected normal-metal samples, with period $h / 2 e$, have been observed in a number of experiments on long cylinders and large 2D arrays of loops. ${ }^{1-3}$ These experiments are in good agreement with the original theoretical predictions of Al'tshuler, Aronov, and Spivak (AAS) ${ }^{4}$ who applied standard ensemble-impurity-averaging techniques (known to give the correct weak-localization results) to a long cylinder. AAS also predicted that similar $h / 2 e$-periodic resistance oscillations would be seen in a single ring. However, more recent theoretical work has clearly shown that in one-dimensional rings, the fundamental period of the oscillation of the quantum mechanical transmission coefficient should be $\Phi_{0}=h / e$, the usual Aharonov-Bohm period. 5.6 This has been confirmed in recent experiments on single rings, ${ }^{7}$ and both $h / e$ oscillations and the AAS effect have been seen in single rings. ${ }^{8}$ The amplitudes of both oscillations decrease exponentially with the ratio $L_{\phi} / P$, where $L_{\phi}$ is the distance over which the electrons maintain their phase coherence, and $P$ is the circumference of the cylinder or ring.

One very important difference has emerged between these two theoretical approaches. ${ }^{9-11}$ The phase of the $h / e$ oscillations in rings with finite width is not fixed relative to zero magnetic field; the phase at $H=0$ is sample specific. The magnitude of the phase depends upon the microscopic details of the impurity configuration. In contrast, the AAS $h / 2 e$ resistance oscillations must always have a maximum (minimum in the presence of strong spin-orbit scattering) at zero field. This difference in zero-field phase dependence between the two theories has been used to explain why $h / e$ oscillations have not been observed in any of the experiments on samples where at least one dimension has been much longer than the phase coherence length (long cylinders or large arrays of loops). In these samples, the $h / e$ oscillations will average to zero since the phases at $H=0$ are uncorrelated in different regions of the sample. Clearly, the $h / 2 e$ oscillations will not average to zero since the phase at $H=0$ is everywhere the same.

We have performed detailed experiments designed to test for the stochastic averaging of the $h / e$ oscillations by the systematic measurement of the amplitude of these oscillations as a function of the number of rings in the limit where the phase coherence length is longer than the diameter of an individual ring but short enough to insure that interference effects involving more than one loop are negligible. We measure both the $h / e$ and AAS $h / 2 e$ oscillations in samples consisting of $N$ rings connected in series and find clear evidence that averaging leads to a $N^{-1 / 2}$ dependence of the amplitude of the $h / e$ oscillations while the amplitude of the AAS oscillations is independent of the number of rings. ${ }^{11}$ Also, the magnitude of the $h / e$ conductance oscillations is in good agreement with recent theoretical ideas on universal conductance fluctuations, ${ }^{12}$ once the ensemble averaging and effects of finite temperature ${ }^{11,13}$ are included.

Our samples were fabricated on $\mathrm{Si}_{3} \mathrm{~N}_{4}$ window substrates by means of conventional electron-beam lithography and liftoff techniques. The Ag films were evaporated from a $99.9999 \%$-pure source. The samples consisted of one, three, ten, and thirty lithographically identical square loops connected in series by line segments of the same width and length as those forming the loops. A transmission electron micrograph of the three-loop sample is shown in Fig. 1(a). The line segments forming the samples were approximately $75 \mathrm{~nm}$ wide, $0.94 \mu \mathrm{m}$ long, and $20 \mathrm{~nm}$ thick. Resistances were measured by standard low-frequency, fourterminal techniques. The sheet resistance of all our samples at $4.2 \mathrm{~K}$ was $R_{\square}=1.47 \Omega / \square$.

Typical data demonstrating both the high- and the low-field magnetoresistance of the single loop and the thirty loops in series at $T=0.32 \mathrm{~K}$ are shown in Fig. 1(b). In both samples, resistance oscillations $h / 2 e$ periodic in the flux are present at low fields superim- 
posed upon a positive magnetoresistance background. Both this positive background and the minimum in the $h / 2 e$ oscillations at $H=0$ indicate that the spin-orbit scattering is strong in our $\mathrm{Ag}$ samples. The amplitude of these $h / 2 e$ oscillations decreases with increasing magnetic field in agreement with the predictions of AAS and with previous experiments on long cylinders $^{1,2}$ and large arrays of rings ${ }^{2,3}$ as well as more recent, single-ring experiments. ${ }^{8}$ At larger magnetic fields, the coherent backscattering has been destroyed, ${ }^{2}$ and the $h / e$ oscillations dominate. The oscillations are superimposed on a random function of magnetic field, the aperiodic fluctuations. ${ }^{13,14}$ Also, the amplitude of the oscillations is modulated by another random function of field. Both of these effects have been previously observed. $7,8,15$
Figure 1 (c) contains the Fourier transforms of the data displayed in Fig. 1(b). The low-field single-ring transforms clearly reveal the presence of a smaller $h / e$ contribution to the spectrum of resistance oscillations. For the thirty-ring, low-field data, the magnitude of the $h / e$ contribution to the observed spectrum is much smaller. This reduction in amplitude of the $h / e$ contribution to the spectrum as the number of series rings increases is also seen in the high-field data.

One necessary condition for obtaining the maximum $h / e$ amplitude in a single ring is that the phase coherence length must be comparable to the perimeter $P$ of the loop. We can obtain an excellent estimate of this parameter by fitting the thirty-loop low-field data by the AAS theory (including paramagnetic and spin-orbit interactions), 3,4

$$
\frac{\Delta R}{R}=\frac{e^{2}}{\pi \hbar} \frac{R_{\square}}{w} \sum_{m=1}^{\infty}\left[3 L_{1} e^{-m\left(P / L_{1}\right)}-L_{0} e^{-m\left(P / L_{0}\right)}\right] \cos \frac{2 \pi m \Phi}{h / 2 e},
$$

where $w$ is the width of the wires, $P$ is the perimeter of the ring, $L_{0}^{-2}=L_{\text {in }}^{-2}+2 L_{S}^{-2}+L_{H}^{-2}, L_{H}^{2}=3(\hbar /$ $e H w)^{2}$, and $L_{1}^{-2}=L_{\text {in }}^{-2}+\frac{4}{3} L_{S_{O}}^{-2}+\frac{2}{3} L_{S}{ }^{-2}+L_{H}^{-2}$. The subscripts refer to inelastic (in), spin-orbit (SO), and spin-flip $(S)$ scattering lengths. We have used the one-dimensional limit of the weak-localization theory ${ }^{16}$ to take into account the uniform background magnetoresistance of the wires. The dash-dotted line in Fig. 1(b) results from the following scattering lengths: $L_{\text {SO }}=0.47 \mu \mathrm{m}, L_{S}=3.1 \mu \mathrm{m}$, and $L_{\phi}=L_{0}(H=0)$ $=2.2 \mu \mathrm{m}$. It should be noted that fitting the background magnetoresistance (exclusive of the oscillations) from $-0.2 \mathrm{~T}$ to $+0.2 \mathrm{~T}$ results in a value $L_{\phi}=2.0 \mu \mathrm{m}$. The different value of $L_{\phi}$ obtained from fitting the AAS oscillations may result from the imperfect fit which results from aperiodic conductance fluctuations and possibly from the fact that the line segments connecting the rings are shorter than $L_{\phi} \cdot{ }^{17,18}$ At $0.3 \mathrm{~K}$, a nearly identical background resistance is also present in single Ag lines $0.075 \mu \mathrm{m}$ wide and 3.0 $\mu \mathrm{m}$ long and the value of $L_{\phi}$ was also found to be within $20 \%$ of $2.0 \mu \mathrm{m}$. For the thirty-ring sample at $T=4.2 \mathrm{~K}$, a single value of $L_{\phi}=1.1 \mu \mathrm{m}$ (and the same values of $L_{S O}$ and $L_{S}$ ) gave an excellent fit to the data over the entire range $-0.2 \mathrm{~T}<H<0.2 \mathrm{~T}$.

At low temperatures the value of $L_{\phi}$ becomes comparable to half the perimeter of the loop, but is still too small to coherently couple more than one loop. While the appropriate phase coherence length for the $h / e$ oscillations may not be $L_{0}(H=0)$ since spin-flip scattering may not destroy the $h / e$ oscillations, ${ }^{11,12,19}$ it is certainly a lower bound. Also, our Fourier analysis clearly detects a small harmonic content in both the $h / e$ and $h / 2 e$ oscillations. In the low-field, thirty-loop data of Fig. 1(b), there is a peak at $h / 4 e$ which corresponds to the direct observation of the $m=2$ term in
Eq. (1).

The models which suggest that $h / e$ will be the fundamental period of oscillation ${ }^{5,6}$ are confirmed again here. As predicted, ${ }^{11}$ all of the samples studied have exhibited $h / e$ oscillations which dominate everywhere except at very low field where the weak-localization corrections (AAS oscillations) are large. At higher fields the $h / e$ oscillations are always larger than the $h / 2 e$ component. When these models are combined with ensemble averaging, they explain the present experiment quite well. A summation of the $h / e$ Aharonov-Bohm effects from uncorrelated spatial regions (which have uncorrelated oscillation phases) causes the overall amplitude of these oscillations to approach zero. As noted above, any effects which are correlated among different regions of the sample will survive the averaging. The $h / e$ oscillations should be uncorrelated between loops. 9,11 This is clearly demonstrated in Fig. 2(a) where we have isolated the $h / e$ oscillations for each of the samples studied here by digitally filtering out all frequencies outside the expected range for $h / e$ periods [determined from the measured inside and outside areas of the ring and shown by the arrows in Fig. 1(c)] and then inverting the transform. The $H=0$ point has been pinpointed in each case by the lowest minimum in the AAS oscillations. Two of the samples $(N=1$ and $N=30)$ have resistance maxima at $H \simeq 0$ and the other two samples have minima. The same absence of correlation should exist between the individual loops in a particular sample. If these four magnetoresistances were summed (as if they were segments of a longer sample) then clearly the average amplitude of the $h / e$ oscillation would decrease.

If the averaging of the uncorrelated oscillations is stochastic $^{9,11}$ the amplitude of the oscillations should 
be proportional to $N^{-1 / 2}$. To test this prediction, we plot the root-mean-square (rms) maximum amplitude of the $h / 2 e$ oscillations at low field (the correlated AAS oscillations) and the $h / e$ oscillations at high field as a function of the number of loops in Fig. 2(b). The $h / e$ amplitudes are calculated from the Fouriertransform data over a field range of 0 to $0.3 \mathrm{~T}$ by use of the method described elsewhere. ${ }^{15}$ The data have

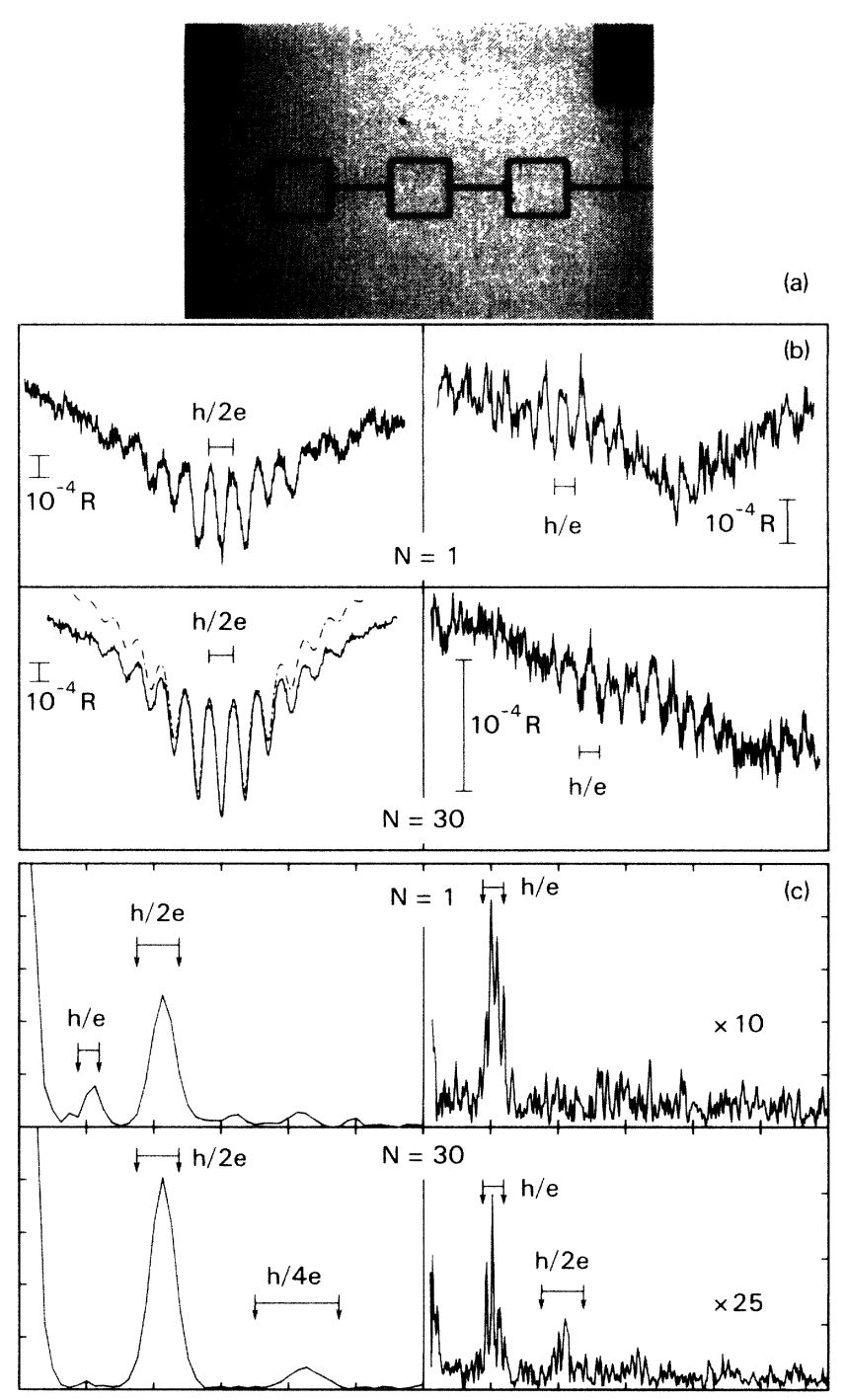

FIG. 1. (a) Transmission electron micrograph of the three-loop sample. (b) Magnetoresistance data at $T=0.32$ $\mathrm{K}$. Clockwise from the lower right-hand corner: the thirtyloop sample for $0.2<H<0.3 \mathrm{~T}$; the thirty-loop sample for $-0.02<H<0.02 \mathrm{~T}$ (the dash-dotted line is the fit by the AAS theory); the single-loop sample for $-0.02<H<0.02$ $\mathrm{T}$; the single-loop sample for $0.15<H<0.25 \mathrm{~T}$. (c) The Fourier transforms of the data in (b). The arrows in the figure indicate the bounds for the flux periods $h / e$ and $h / 2 e$ based on the measured inside and outside areas of the loop. been scaled to account for the wires connecting the loops which yield no oscillations. After this correction, the rms oscillation size is $\Delta G=\Delta R / N R_{L}^{2}$, where $\Delta R$ is the rms value of the resistance change of the sample, and $R_{L}$ is the resistance of one loop without leads. Two observations are immediately made. First, the AAS oscillations are not affected by the averaging of uncorrelated regions. This was expected ${ }^{9,11}$ since these oscillations exist in very large samples. ${ }^{1-3}$ Second, the $h / e$ oscillations die out as the square root of the number of loops. With extrapolation to very large arrays, such as those studied previously, ${ }^{2,3}$ they would be buried in the noise.

The absolute size of both the $h / e$ and $h / 2 e$ oscillations are predicted ${ }^{11,12}$ to be of the order of the fundamental unit of conductance $\left(G_{0}=e^{2} / h\right)$ when the inelastic processes and the thermal smearing are negligible. Energy averaging, due to thermal smearing, is a source of self-averaging which applies when uncorrelated conduction states in one of the loops contribute
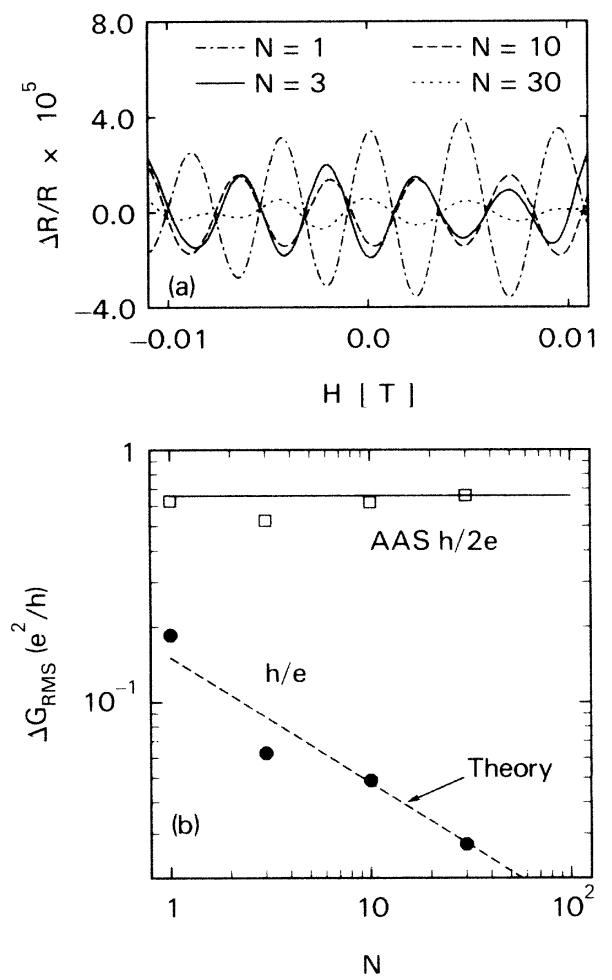

FIG. 2. (a) The $h / e$ oscillations near zero field for the four samples extracted from the raw data by a digital filter set to include all periods allowed by the measured inside and outside areas. (b) The rms conductance amplitude of the $h / e$ (circles) and the $h / 2 e$ (squares) oscillations as a function of the number of loops in the sample. The dashed line represents the calculated amplitude of the $h / e$ oscillations, and the solid line is the theoretical value for the $h / 2 e$ oscillations predicted by AAS for $L_{\phi}=2.2 \mu \mathrm{m}, L_{S O}=0.47 \mu \mathrm{m}$, and $L_{S}=3.1 \mu \mathrm{m}$. 
to the fluctuations in the conductance. ${ }^{13}$ The number of uncorrelated conduction patterns is $n \simeq k_{\mathrm{B}} T / E_{c}$, where $E_{c}=2\left(G / G_{0}\right) \Delta E$, and $\Delta E$ is the level separation in the sample. ${ }^{11,13}$ For our loops, $E_{c} \simeq 0.046 \mathrm{~K}$ implying that $n=7$ in each loop at $T=0.3 \mathrm{~K}$. The dashed curve in Fig. 2(b) represents $0.4 G_{0} / N_{\text {eff }}^{1 / 2}$, where $N_{\text {eff }}=n N$, the number of patterns times the number of loops. ${ }^{11}$ This assumes that both energy averaging and ensemble averaging are independent processes. ${ }^{11}$ Our data are in excellent agreement with theoretical calculations. $^{11,12}$ The theory of AAS for $L_{\phi}=2.2 \mu \mathrm{m}$ has been used to calculate the amplitude of the $h / 2 e$ oscillations, and is shown by the solid line in Fig. 2(b).

In summary, experiments on series arrays of one, three, ten, and thirty normal-metal loops have confirmed that averaging of uncorrelated regions of the sample results in a $N^{-1 / 2}$ amplitude dependence of the $h / e$ Aharonov-Bohm oscillations, but does not reduce the $h / 2 e$ oscillations that result from coherent backscattering. It is this averaging which is responsible for failure to observe the $h / e$ period in the experiments on large samples (cylinders and arrays of rings). The amplitudes of the $h / e$ and $h / 2 e$ components of the magnetoresistance are consistent with recent theoretical work on universal conductance fluctuations including both energy-averaging and ensemble-averaging mechanisms in the samples.

The authors wish to thank S. Rishton, H. Luhn, and D. Kern for useful advice and assistance in the sample fabrication. We also benefitted from many discussions with M. Büttiker, Y. Gefen, Y. Imry, R. Landauer, P. Santhanam, and A. D. Stone. One of us (C.V.H.) is Bevoegdverklaard Navorser of the National Fonds voor Wetenschappelyk Onderzoek, Belgium.

(a) Permanent address: Laboratorium voor Vaste StofFysika en Magnetisme, University of Leuven, B-3030 Leuven, Belgium.

${ }^{1}$ D. Yu. Sharvin and Yu. V. Sharvin, Pis'ma Zh. Eksp.
Teor. Fiz. 34, 285 (1981) [JETP Lett. 34, 272 (1981)].

${ }^{2}$ See the review by $\mathrm{Yu}$. V. Sharvin, Physica (Amsterdam) $126 \mathrm{~B}+\mathrm{C}, 288$ (1984).

${ }^{3}$ G. J. Dolan, J. C. Licini, and D. J. Bishop, to be published; D. J. Bishop, J. C. Licini, and G. J. Dolan, Appl. Phys. Lett. 46, 1000 (1985).

${ }^{4}$ B. L. Al'tshuler, A. G. Aronov, and B. Z. Spivak, Pis'ma Zh. Eksp. Teor. Fiz. 33, 101 (1981) [JETP Lett. 33, 94 (1981)].

${ }^{5}$ M. Büttiker, Y. Imry, and R. Landauer, Phys. Lett. 96A, 365 (1983); Y. Gefen, Y. Imry, and M. Ya. Azbel', Surf. Sci. 142, 203 (1984), and Phys. Rev. Lett. 52, 129 (1984); M. Büttiker, Y. Imry, and M. Ya. Azbel', Phys. Rev. A 30, 1982 (1984)

${ }^{6}$ M. Büttiker, Y. Imry, R. Landauer, and S. Pinhas, Phys. Rev. B 31, 6207 (1985).

${ }^{7}$ R. A. Webb, S. Washburn, C. P. Umbach, and R. B. Laibowitz, Phys. Rev. Lett. 54, 2696 (1985).

${ }^{8}$ V. Chandrasekar, M. J. Rooks, S. Wind, and D. E. Prober, Phys. Rev. Lett. 55, 1610 (1985).

${ }^{9} \mathrm{Y}$. Gefen, private communication; M. Murat, Y. Gefen, and Y. Imry, unpublished.

${ }^{10}$ D. A. Browne, J. P. Carini, K. A. Muttalib, and S. R. Nagel, Phys. Rev. B 30, 6798 (1984).

${ }^{11}$ A. D. Stone and Y. Imry, Phys. Rev. Lett. 56, 189 (1986).

12P. A. Lee and A. D. Stone, Phys. Rev. Lett. 55, 1622 (1985).

${ }^{13}$ A. D. Stone, Phys. Rev. Lett. 54, 2692 (1985).

${ }^{14}$ R. A. Webb, S. Washburn, C. P. Umbach, and R. B. Laibowitz, in Localization, Interaction, and Transport Phenomena in Impure Metals, edited by G. Bergmann, Y. Bruynseraede, and B. Kramer (Springer-Verlag, Berlin, 1984), p. 121; C. P. Umbach, S. Washburn, R. B. Laibowitz, and R. A. Webb, Phys. Rev. B 30, 4048 (1984).

${ }^{15}$ S. Washburn, C. P. Umbach, R. B. Laibowitz, and R. A. Webb, Phys. Rev. B 32, 4789 (1985).

16J. C. Licini, G. J. Dolan, and D. J. Bishop, Phys. Rev. Lett. 54, 1585 (1985).

${ }^{17}$ B. Douçot and R. Rammal, Phys. Rev. Lett. 55, 1148 (1985).

${ }^{18}$ B. L. Al'tshuler, A. G. Aronov, and A. Yu. Zyuzin, Zh. Eksp. Teor. Fiz. 86, 709 (1984) [Sov. Phys. JETP 59, 415 (1984)]

${ }^{19}$ B. L. Al'tshuler, Pis'ma Zh. Eksp. Teor. Fiz. 41, 530 (1985) [JETP Lett. 41, 648 (1985)]. 


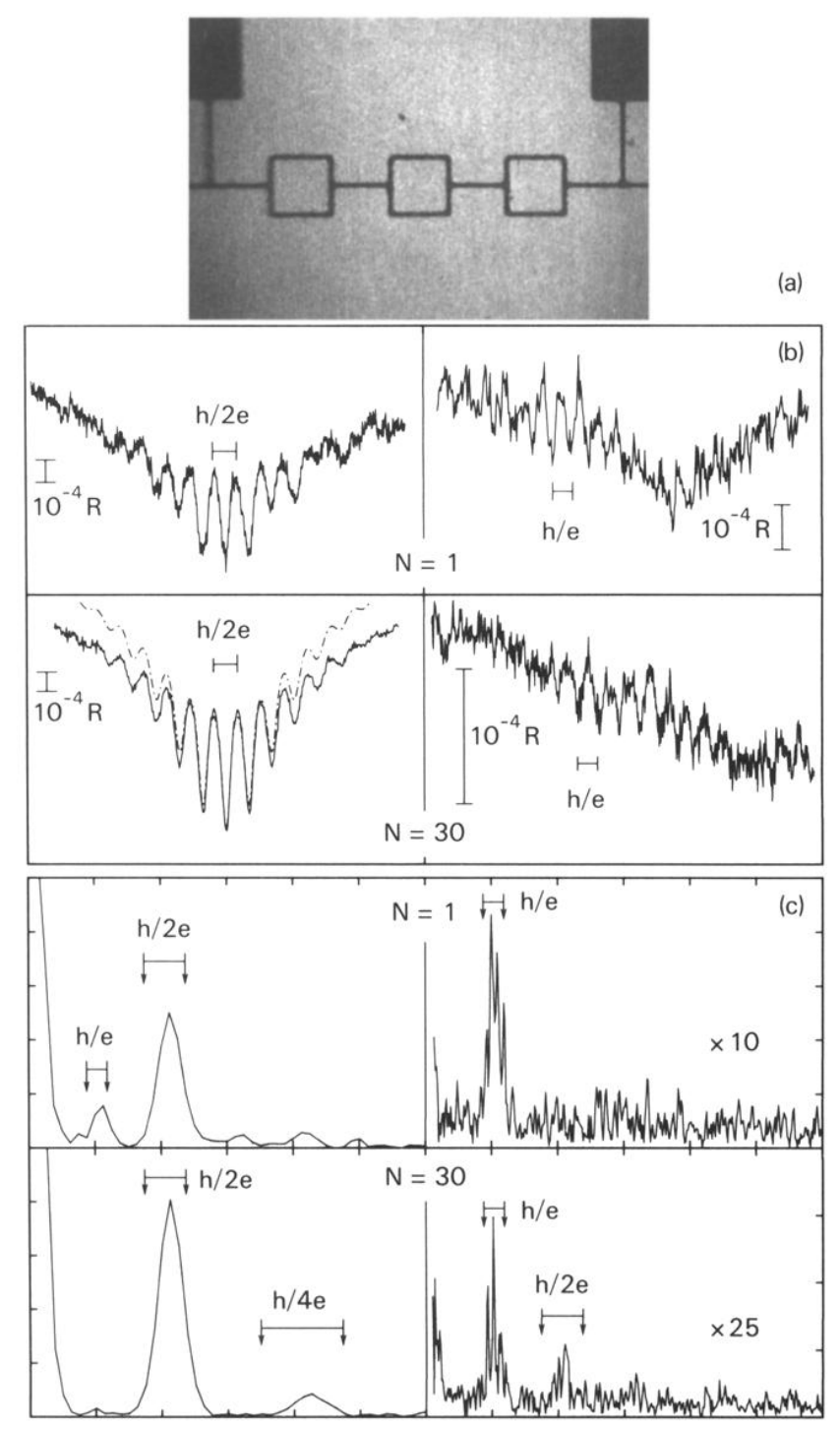

FIG. 1. (a) Transmission electron micrograph of the three-loop sample. (b) Magnetoresistance data at $T=0.32$ K. Clockwise from the lower right-hand corner: the thirtyloop sample for $0.2<H<0.3 \mathrm{~T}$; the thirty-loop sample for $-0.02<H<0.02 \mathrm{~T}$ (the dash-dotted line is the fit by the AAS theory); the single-loop sample for $-0.02<H<0.02$ $\mathrm{T}$; the single-loop sample for $0.15<H<0.25 \mathrm{~T}$. (c) The Fourier transforms of the data in (b). The arrows in the figure indicate the bounds for the flux periods $h / e$ and $h / 2 e$ based on the measured inside and outside areas of the loop. 